\title{
The Coastal Currents Offshore the Nuclear Power Plants at Northern Taiwan
}

Jian-Hwa Hu

Department of Marine Environmental Informatics, National Taiwan Ocean University, Keelung, Taiwan, R.O.C., hujh@mail.ntou.edu.tw

Follow this and additional works at: https://jmstt.ntou.edu.tw/journal

Part of the Terrestrial and Aquatic Ecology Commons

\section{Recommended Citation}

Hu, Jian-Hwa (2004) "The Coastal Currents Offshore the Nuclear Power Plants at Northern Taiwan," Journal of Marine Science and Technology. Vol. 12: Iss. 5, Article 1.

DOI: $10.51400 / 2709-6998.2256$

Available at: https://jmstt.ntou.edu.tw/journal/vol12/iss5/1

This Research Article is brought to you for free and open access by Journal of Marine Science and Technology. It has been accepted for inclusion in Journal of Marine Science and Technology by an authorized editor of Journal of Marine Science and Technology. 


\title{
THE COASTAL CURRENTS OFFSHORE THE NUCLEAR POWER PLANTS AT NORTHERN TAIWAN
}

\author{
Jian-Hwa $\mathrm{Hu}$
}

Key words: coastal tidal currents, ADCP, synoptic, vortices.

\begin{abstract}
Coastal tidal currents play an important role to disperse the warm waters outlet from cooling system of a nuclear power plant (NPP). The modern technique of ship-board acoustic Doppler current profiler (SB-ADCP) was intensively applied to scan the current fields around the three NPPs at northern Taiwan. Sophisticated data processing provides time series of synoptic flow charts of vectors in grids associated with streamlines, allowing the visualization of how the bay like coastline input its boundary layer effect to the current field. The generation of cyclonic or anti-cyclonic vortices inside the bay in flood or ebb tide and their migration to out of the bay later on to participate the alternation of the tidal currents are remarkably observed. The difference among the three bay-like coasts or bottom topography in shape or size should be the main cause to the different behaviors of their vortices, emphasizing the variety of the dynamic backgrounds for the environmental studies upon the thermal plumes.
\end{abstract}

\section{INTRODUCTION}

Normally coastal currents are mainly composed of tidal components. It has been well known that tides along the coast of northern Taiwan are in mixed type of diurnal and semi-diurnal periods, in which the latter plays a major role in general. Furthermore, the tides initiate at the east coast of Taiwan propagating counterclockwise to pass over the north coast and then enter the Taiwan Strait. Therefore, the coastal currents are characterized by the backwards and forwards variation of tidal flows with the same periods. At northern Taiwan, the general orientation of the coast is about NW-SE, guiding the coastal NW currents in flood tide and SE currents in ebb tide as shown in Fig. 1. However, due to the coastal boundary layer effect (Hu, 2004), currents within certain near-shore range may behave not only asynchronous with those of offshore regions but also in complex flow patterns. For instance, the near shore

Paper Submitted 11/03/04, Accepted 11/30/04. Author for Correspondence: Jian-HwaHu.E-mail: hujh@mail.ntou.edu.tw.

*Department of Marine Environmental Informatics, National Taiwan Ocean University, Keelung, Taiwan, R.O.C. coastal currents crossing over a sill right outside the Keelung Harbor were far beyond what one would have imagined before careful surveys were done (Hu, 2001a).

Human beings continue to utilize and rely on the near shore waters, being more concern about the coastal environment conditions. The currents under tide motions construct the main dynamic field for various pollutants to advectively disperse or transport over a wide range, while waves might significantly cause vertical mixing in relatively smaller water area. Nuclear power plants would generate considerable amount of warm waters for cooling purposes. The heat released to coastal regions since ever is thought to be a major factor of inducing any other variations of environmental indices if radioactive materials do not input there. In northern Taiwan, there are two nuclear power plants (named as NPP1 and NPP2 in this article) having been running for more than twenty years. After the third plant established at southern Taiwan, the fourth is still under construction at north again and named as NPP4. The three plants at north of Taiwan are marked in the map of Fig. 1.

Since the cooling water of power plants also comes from the near shore in the neighborhood, the higher

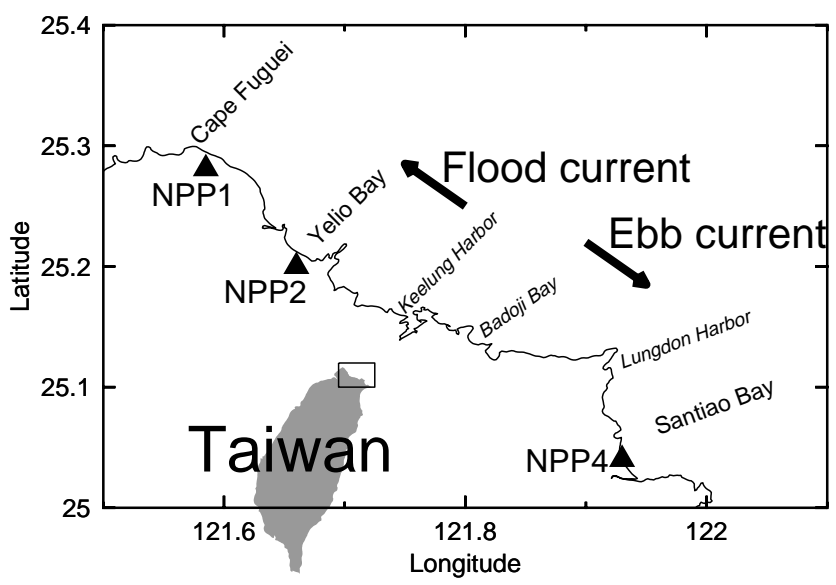

Fig. 1. General offshore tidal currents at northern Taiwan. The three nuclear power plants NPP1, NPP2 and NPP4 are marked by $\triangle$. 
temperature would make the thermal plume of the same water with lower density and hence quite thin at surface to spread out almost right outside the discharged channel. To understand how the surface plume shifted, shaped or distorted by the coastal currents becomes a background study of the entire environmental surveys although the along-with temperature distribution must be another topic. However, comparing to the big mass of the sea waters near the plant, the discharged mass of the thermal plume should not be a cause of the coastal current variations or part of its characteristics except where of very surface and at the outlet. On the contrary, the coastal currents would substantially influence the thermal plume, thereafter what are they by all means becomes the first question to be answered.

\section{OBSERVATIONS AND DATA PROCESSING}

In classical fluid dynamics, currents may be observed in either Eulerian or Lagrangian methods. Mooring current meters provides information in the former manner, while tracking free drifters delineates a flow in later mode. These two are sometimes employed together to remedy their respective deficiency in time or space domain when their individual theories and practical approaches have been well developed for century long. Even though, such methods are still constrained by unavoidable limited capability. The amount of information may not be greatly enhanced due to the high costs and difficulties of equipments and their conductions especially for ocean purposes.

In the past decades, a modern technique was developed in a great progress for current observation according to the Doppler effects of acoustic signals backscattered by suspended particles in moving waters. It was thereby termed as the ADCP, the acronym of Acoustic Doppler Current Profiler, providing current velocities of various layers of a water column as from a system of many current meters attached to a vertical wire. Namely, it can be also moored as the Eulerian method. However, more recent skills install the system to a moving vessel (termed as ship-board or SB-ADCP) for two or three dimensional currents scanning purposes depending upon the cruise track over just one line or few lines covering a water area. A problem arises then that such a scanning method is neither Eulerian nor Lagrangian since the SB$\mathrm{ADCP}$ is not in free drifting, hence requesting a new logistics to understand the observed data in space domain but with time lags.

Mostly, utilities built at coast would select a bay area for better sea states in most of time in order to protect the construction. The contradiction must be that the thermal plume of power plant requires a more dynamic coastal environment to diffuse efficiently while severe sea conditions are usually happened to a cape or rather opened coast line. Fig. 1 shows the NPP1 located near Cape Fuguei, the northern tip of Taiwan and famous for its rough sea states. In fact, there is a bank of reefs about five hundred meters away from the coast between the NPP1 and the Cape, forming a small semibay condition with the breakwater at east of the plant (Fig. 2). On the other hand, the NPP2 resides far inside the C-type Yelio Bay and so as for the NPP4 in the Ltype Santiao Bay (Fig. 1). In his early studies, $\mathrm{Hu}$ (2001b) pointed out that, under the regular coastal tidal currents outside the Badoji Bay (Fig.1), the flows inside the bay were too complicated to be well understood with both of Eulerian and Lagrangian observations. Accordingly, the SB-ADCP was considered to improve the current field observations in the coastal regions of the three plants.

Considering the dominant period of semi-diurnal tide, the SB-ADCP scanning should last at least for twelve hours, if the two- or three-dimensional flow pattern over the region is thought to be more intrinsic than the mean flow variation in long term for the plume study. Nevertheless, the diurnal tide cannot be ignored and a complete cycle of semi-diurnal tidal currents should be followed, demanding a relevant cruise being at least twenty-four hours for each survey. Also because of the semi-diurnal period, each sampling should be better less than a certain time interval (of course the shorter the better) to give appropriate description for a harmonic tidal signal. However, to the contrary, the sampling time interval limits the area a pass of scanning can be finished over it. An optimum choice of two to three hours could be empirically set. Then few passes of

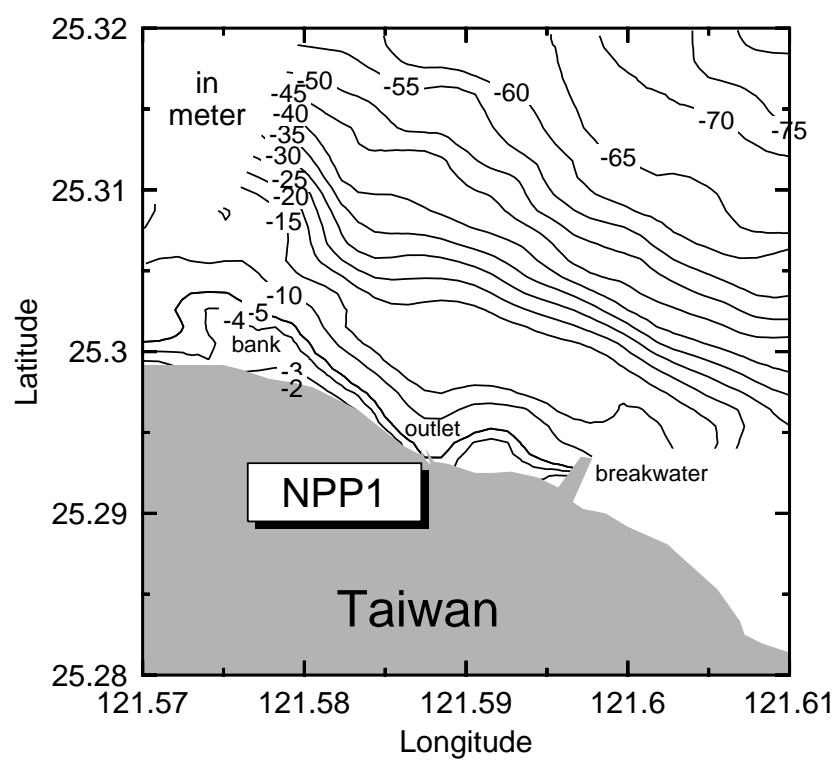

Fig. 2. Bottom topography near the NPP1. 
the repeated scanning per twenty-four hours may successfully reveal the complex of the very coastal current field when the few cruise lines covering the field are properly designed with the compromise between the pass time interval and the ship speed for better performance according to the type of the SB-ADCP.

Notwithstanding, within a pass of two to three hours scanning, the time lag problem still exists when the tide motion proceeds. About ten passes of scanning during a day thus allows a suitable interpolation of current velocities along the cruise lines for any selected time of the day. Then a time series, say hourly, of synoptic charts in grids of the current field can be obtained by general objective analysis. With this Eulerian format of velocity measurements, further works may be done straightforward, such as the stream functions and others of oceanic velocity gradients in kinematic derivative properties ( $\mathrm{Hu}, 1983)$.

Recently another SB-ADCP data processing method was proposed by Candela et al. (1992) to decompose all scanning data via a linear regression of harmonic equation into various tidal components and the residual out of the mean current at selected nodes in the field. And then the objective analysis can be applied to contribute those tidal components as ellipses and the means at nodes to all grids. After that, Chen (2002) summed up all in respective times for individual grids to be a time series of Eulerian flow charts but without the residuals. The results were found so closed to what from the synoptic processing introduced above. However, the merit of the harmonic method is that it can be available for an ADCP scan along a track just once without repeat by assuming the characteristic of the tidal wave crossing over the area is about the same at any spots, and therefore significantly enlarges the surveying area. Such an assumption intuitively accepted in open seas might not be adequate in coastal regions where the geography and bottom topography make the propagating tidal wave not similar from place to place.

In this article, the current velocities in grids are derived from those surveys by two devices. The RDI's SB-ADCP mounted on the research vessel "Ocean Researcher II" (RV/OR2) of the National Taiwan Ocean University transmits sound signals of low frequency of $75 \mathrm{MHz}$ to reach a depth of over $500 \mathrm{~m}$ but with a price of large surface blank zone of about $16 \mathrm{~m}$. It was examined that somewhat away from the coast of northern Taiwan, the $16 \mathrm{~m}$ velocities mostly well represent the flows of surface layer above the depth (Chen, 2004). The comparison was made with a high frequency equipment of $750 \mathrm{MHz}$, the SonTek's ADP, towed by a fishing boat during an experiment. The ADP's blank zone of less than $2 \mathrm{~m}$ compensates a small response depth of less than $100 \mathrm{~m}$, satisfying near shore scans.
For the purpose of the synoptic processes, the RV/OR2 or fishing boat steamed 24 hours strictly for each cruise to meet the basic criterion.

As the tidal currents are concerned of, a pressure gauge was launched at bottom near the NPP1 and Npp2 to compare to the interpolation from the tides at northern Taiwan reported by the Central Weather Bureau (ROC-CWB). The results showed a high agreement with a deviation of few minutes and few centimeters in high or low tides (Hu and Fang, 2000) and therefore the interpolated tide information is applied in this article. For the NPP4, the ROC-CWB tides at Lungdon Harbor (Fig. 1) are practically available for our purpose.

\section{CURRENTS AT NPP1}

In Fig. 1, the coastline around NPP1 shows rather convexly opened to the sea. In fact, at east of the discharged channel, a breakwater was built (Fig. 2) to protect the intake system of cooling water. In Fig. 2, bottom topography is also shown in contours, and a subsurface valley right connected to the outlet of the discharged channel can be identified by water depths less than 5-10 m. The east slope of the valley orients quite offshore (i.e., normal to the shore) but does not extend very far, while its west slope elongates towards northwest to a great distance with a small angle gradually away from the coast. The subsurface valley actually is an extension of the valley on land where the NPP1 sits against the severe sea states in winter. The discharged channel was accordingly constructed along the bottom of the coastal valley, rendering the thermal plume west-oriented preferable to the east or normal to the coast. Fig. 3(a) shows the tracks of GPS drifters deployed at the outlet of NPP1's discharged channel near the end of an ebb tide. Namely, the warm water was running merely by following the guidance of the channel at about the slack time of tides.

Notwithstanding, agreeing with the typical northwest flow in flood tide, the case in Fig. 3(b) discloses another feature of near shore effect by the west boundary of the subsurface valley. Namely, a near shore reversed current might have been there and encountered the plume to form the clockwise turning before merging to the NW flood current at far shore. Such a Lagrangian study seemed having proved and explained the current field at NPP1 very clear as many others in the past few years (Hu and Fang, 2000; Hwang et al., 2002).

Nevertheless, the times series of the ADCP synoptic flow charts in Fig. 4 offer a quite different view of the currents from the near field to the far field. The reversions of the tidal currents from ebb to flood and vice versa were actually accomplished by an offshore shifting of the slack zone where the currents almost 
diminished. In other words, the slack zones always initiated at the subsurface valley. As a matter of fact, after a careful inspection of Fig. 4, one would realize the slack zone sometimes seemed to be a vortex in between the two reversed flood tide and ebb tide flows.

To delineate the flow pattern in better visualization manner, Chang (2002) invokes streamlines with arrows showing the along with moving directions as in Fig. 5. It is seen that the first event occurred at 15:00 of the day at coast and shifted at 16:00 offshore, where a vortex was not clearly formed in the alternation of the tidal currents near the high tide. Nevertheless, during

(a)

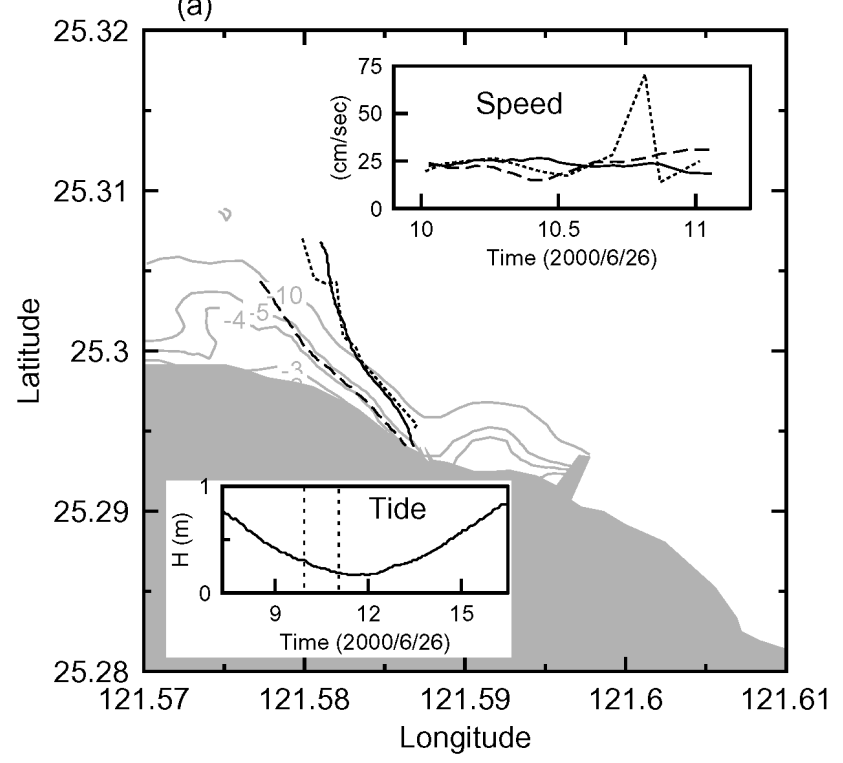

(b)

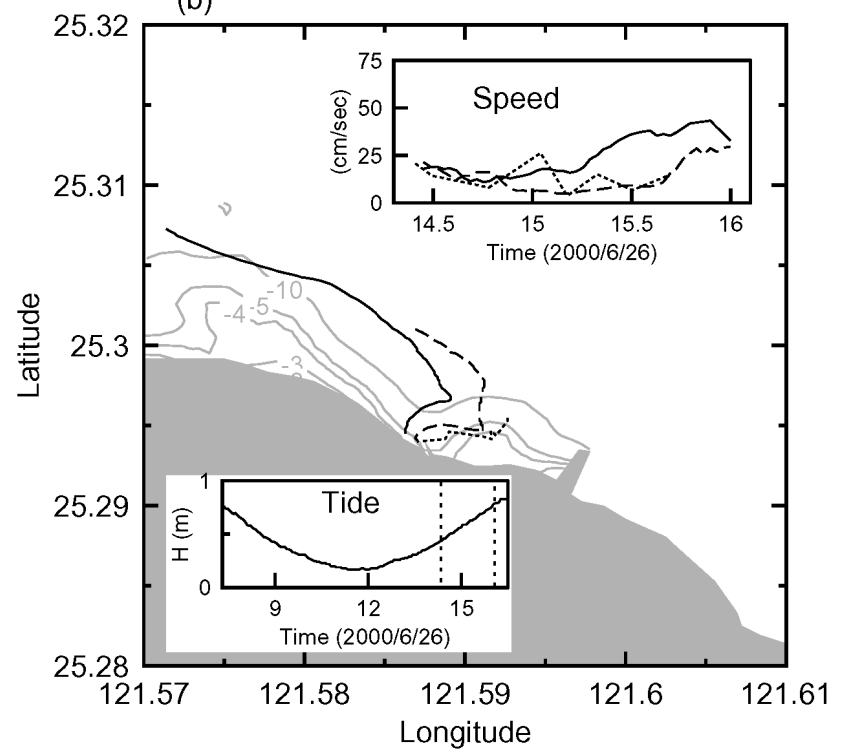

Fig. 3. Trajectories and speeds of 3 GPS drifters deployed at outlet of discharged channel at NPP1 on 2000/6/26 during (a) ebb tide and (b) flood tide. Bottom topography of depth less than $10 \mathrm{~m}$ is illustrated with contours. the ebb tide, an anti-cyclonic vortex started generating on the leeward of the west bank since 18:00 and became a well formed vortex one hour later. Then it moved offshore in a speed of about $2 \sim 3 \mathrm{~km}$ per 5 hours, or roughly $0.5 \mathrm{~km} /$ hour. At 04:00 of the next day (Julian hour 28:00 since 00:00 on 2001/7/27 in Fig. 5), a semi cyclonic gyre developed near the high tide again as that at 15:00 of the previous day evidenced that the phenomenon was repeatable at least for days or maybe the season.

Accordingly, the theme played in Fig. 5 implies the leading of the very coastal water motions to that of the far shore during the tide alternation. The mechanism should be the boundary effect of the coastal geography and bottom topography, rendering the process quite unlikely recognized from observations of current meters moorings, say one at few hundreds meters and the other at $3 \mathrm{~km}$ away from the coast. As to why not an equivalent vortex on the leeward of the breakwater was

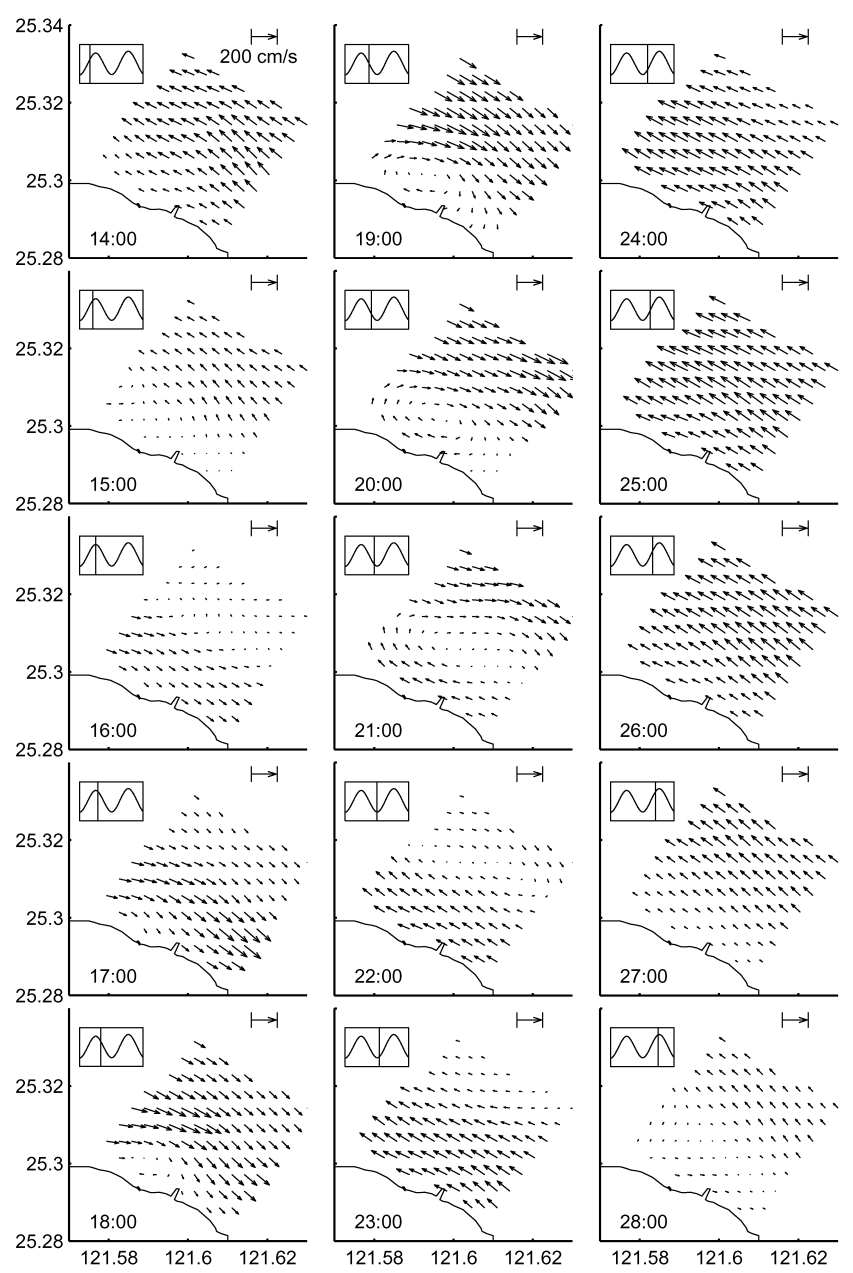

Fig. 4. Synoptic flow charts in vectors from SB-ADCP surveys at NPP1 with respect to local tide. Time of each frame is in Julian hour since 2001/7/27. 
seen in flood tide, the details in Fig. 6 of another ADP shallow water surveys on the same day indeed disclosed the similar actions to form a complete vortex. However, the cyclonic vortex had no chance to move offshore before merging into the bigger vortex from the west (see 15:00 in Fig. 5) due to the small size of the subsurface valley (illustrated by the gray area in Fig. 5) or perhaps the opposition of westward plume of warm waters outgoing thru the channel.

\section{CURRENTS AT NPP2}

As shown in Fig. 7, the NPP2 was built at far inside of Yelio Bay with its discharged channel pointed to the center of the bay. The two capes of the bay are Kinsan on the west and Yelio Peninsula the east, widely opening the bay to the sea. Nonetheless, there are three huge rocks named "Three-Candlesticks" stand upright on the east of Kinsan with their subsurface reef base blocking about one third of the bay mouth. Therefore Yelio Bay
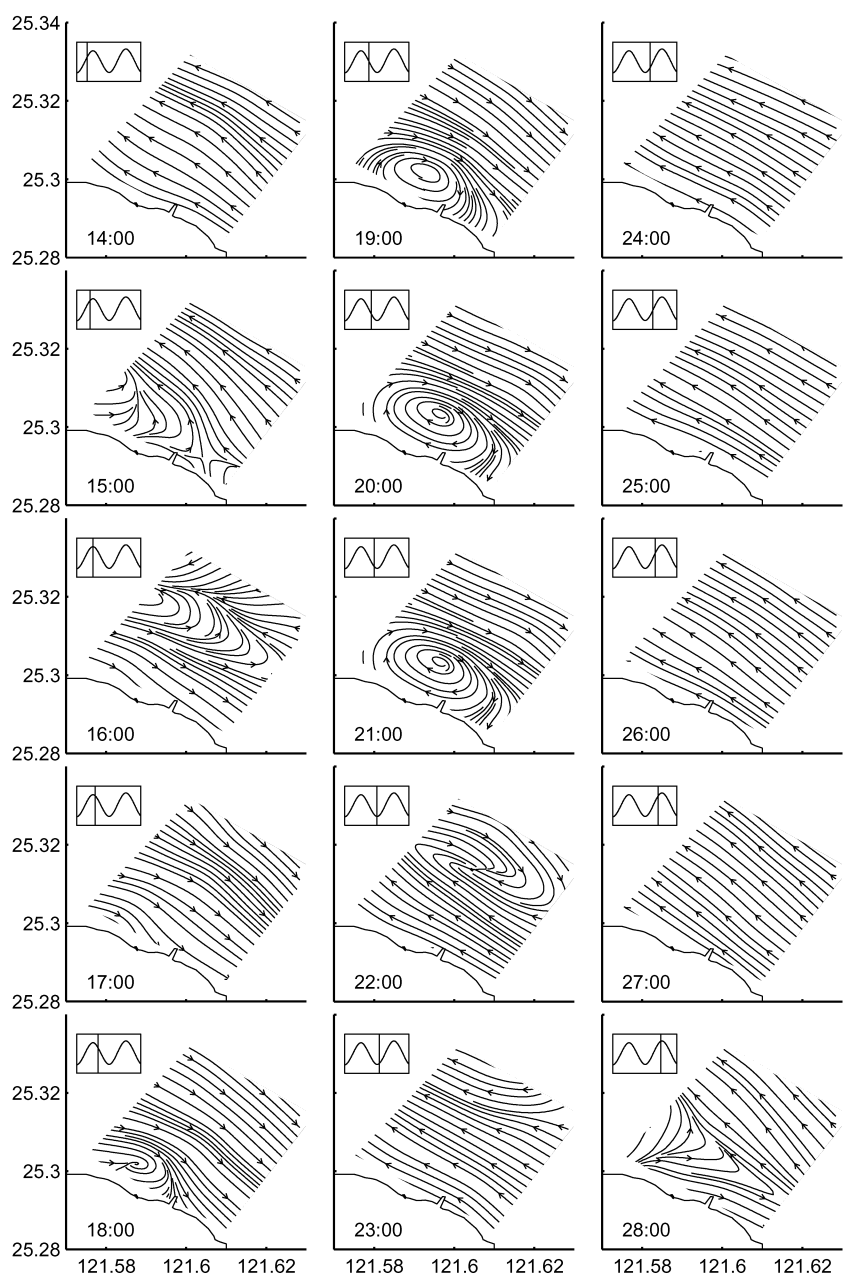

Fig. 5. Synoptic flow charts in streamlines of Fig. 4. may be viewed as a narrow mouth bay, in where the current field was intensively observed by five moorings of current meters and multi-deployments of three to five GPS drifters (Hu and Fang, 2000). Except for that the thermal plume would have been swinging from west to east and vice versa in tide periods, not much else could be detected about how the currents passed through the five moorings anchored along a line away from the discharged channel.

And then, the ADCP surveys were applied instead in the passed few years as for the NPP1. Fig. 8 shows the combination of the current field in vectors at grids as well as streamlines associated. It is clearly seen that currents outside the bay proceeded well in response to tides as for granted, while their alternations between flood and ebb played somewhat differently. The story
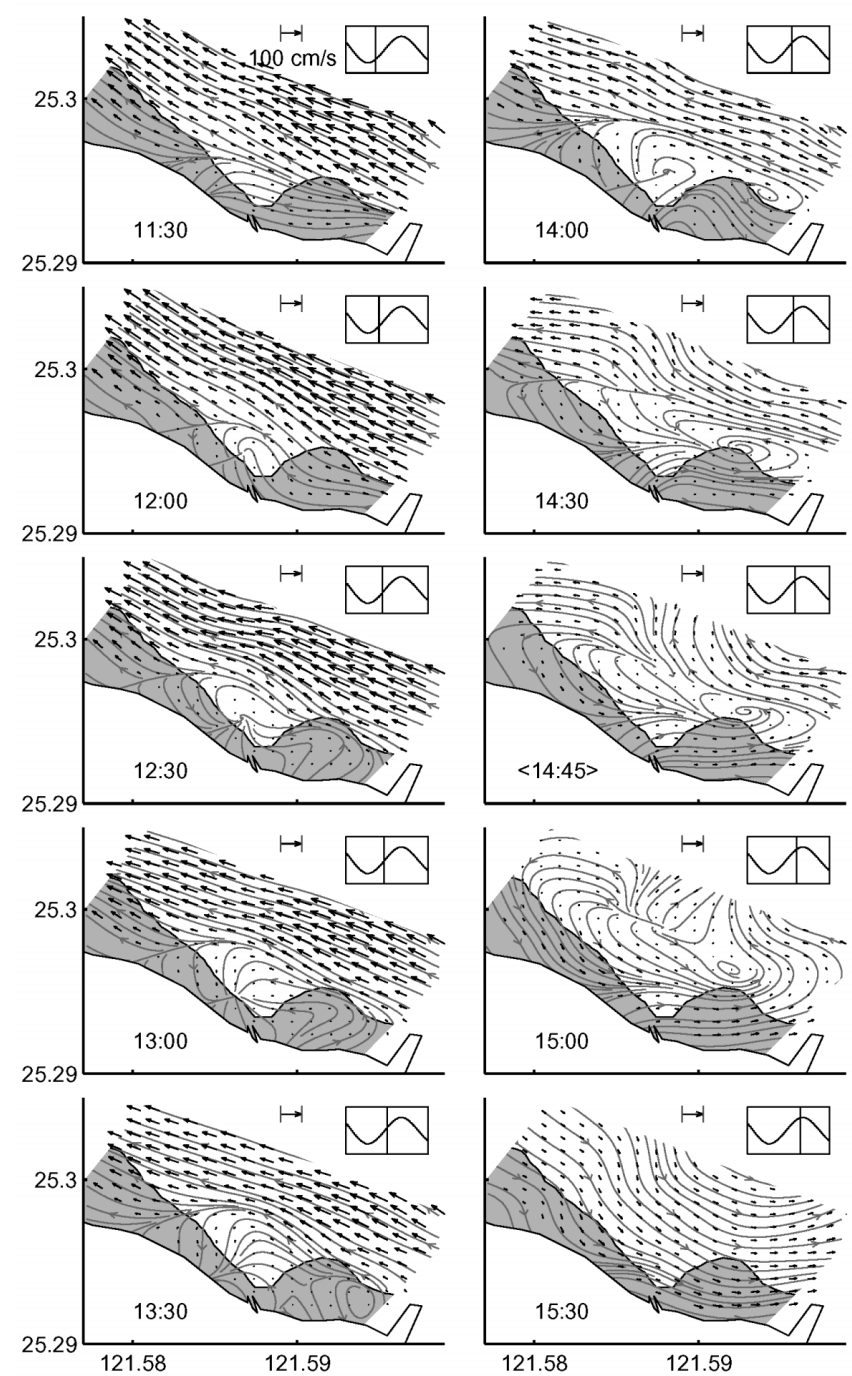

Fig. 6. Near shore synoptic flow charts in vectors and streamlines from ADP surveys at NPP1 on 2001/7/27 vs. local tide. Depth of gray area is less than $5 \mathrm{~m}$. 
might start from 10:30 when the southeast ebb currents at or within the bay mouth hit the west coast of Yelio Peninsula and thereby forced the flow skirting around the bank of Three-candlesticks to intrude the bay near the center of the bay mouth. At 11:30, such an intrusion triggered the formation of an anti-cyclonic gyre at the coast of Yelio. After then, this gyre became mature and bounced out to the northwest from the coast. It seemed decayed in a short time at 14:30, but actually attributed to the clockwise turning of currents outside the bay for the slack sea of low tide at about 16:00. When the turning caused an onshore flow outside the bay before the slack time, a long-shore northwest flow was induced at the bay mouth leading the flood currents of the entire region.

While the westward flood currents established since 16:30 (Fig. 8), similar intrusion to the bay occurred again but inducing two gyres, one for each capes. Must have been due to the difference of geography, the cyclonic gyre generated by the long-extension of Yelio Peninsula was indeed much more rigid than the other by the bank of Three-candlesticks. The latter was so weak to form a complete one, while the Yelio gyre moved away the cape tip and then stayed where facing the center of the bay mouth. It then dissipated before the high tide arrived at 22:30. The following realizations ensured what for ebb tide as discussed above.

As to where in Fig. 8 lacked of current information inside the bay was due to the depths not much greater or even shallower than the $16 \mathrm{~m}$ blank zone of the ADCP system on RV/OR2. Similarly in the field experiment, an ADP daytime survey was simultaneously proceeded over there by a small fishing boat but to provide the synoptic flow charts only for 10:10 14:40 as shown in Fig. 9. Comparing them to those of 10:30 14:30 in Fig. 8 , the cyclonic gyre in ebb caused by the bank of Three-

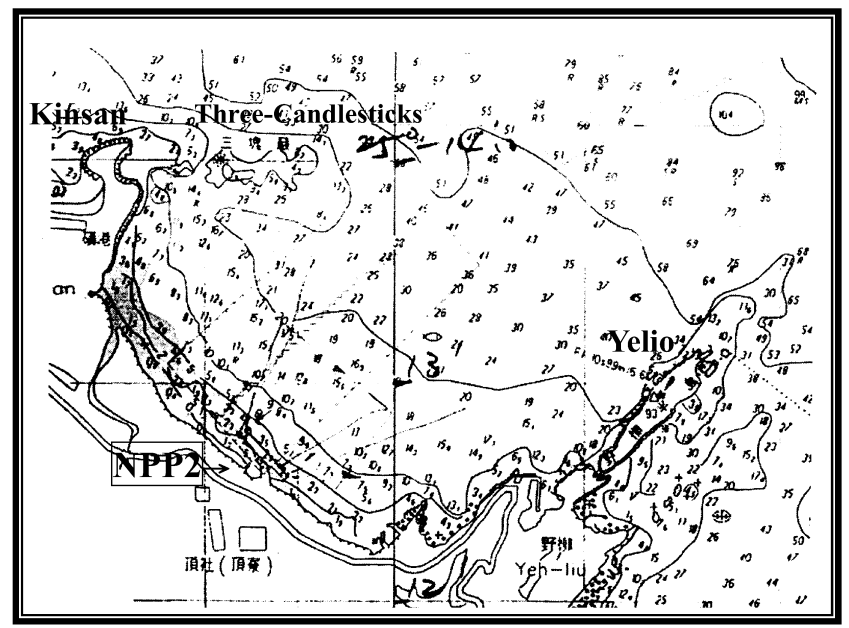

Fig. 7. The bottom topography at Yelio Bay where the NPP2 located. candlesticks was then clearly seen because of the finer scans of the ADP task. But more interesting feature in Fig. 9 must be how the flow of the thermal plume ejected from the discharged channel interacted with the gyre to form a convergent front near the Peninsula. Along the front, white foam and floating trash gathered occasionally as a streak (Hwang et al., 2002).

\section{CURRENTS AT NPP4}

Santiao Bay is the biggest bay in northern Taiwan (Fig. 1). However, the bay shape is far different from the general shape of capital letter C. It looks rather like the letter $\mathrm{L}$ as embedded in gray in Fig. 10, widely opening the bay mouth with the north tip of about $10 \mathrm{~km}$ vertical bar at Bito Cape and the south bar of Santiao Peninsula. The brook Double-creeks discharges its water with abundant suspended particles right at the intersection of the two bars, supplying sand for the $3 \mathrm{~km}$

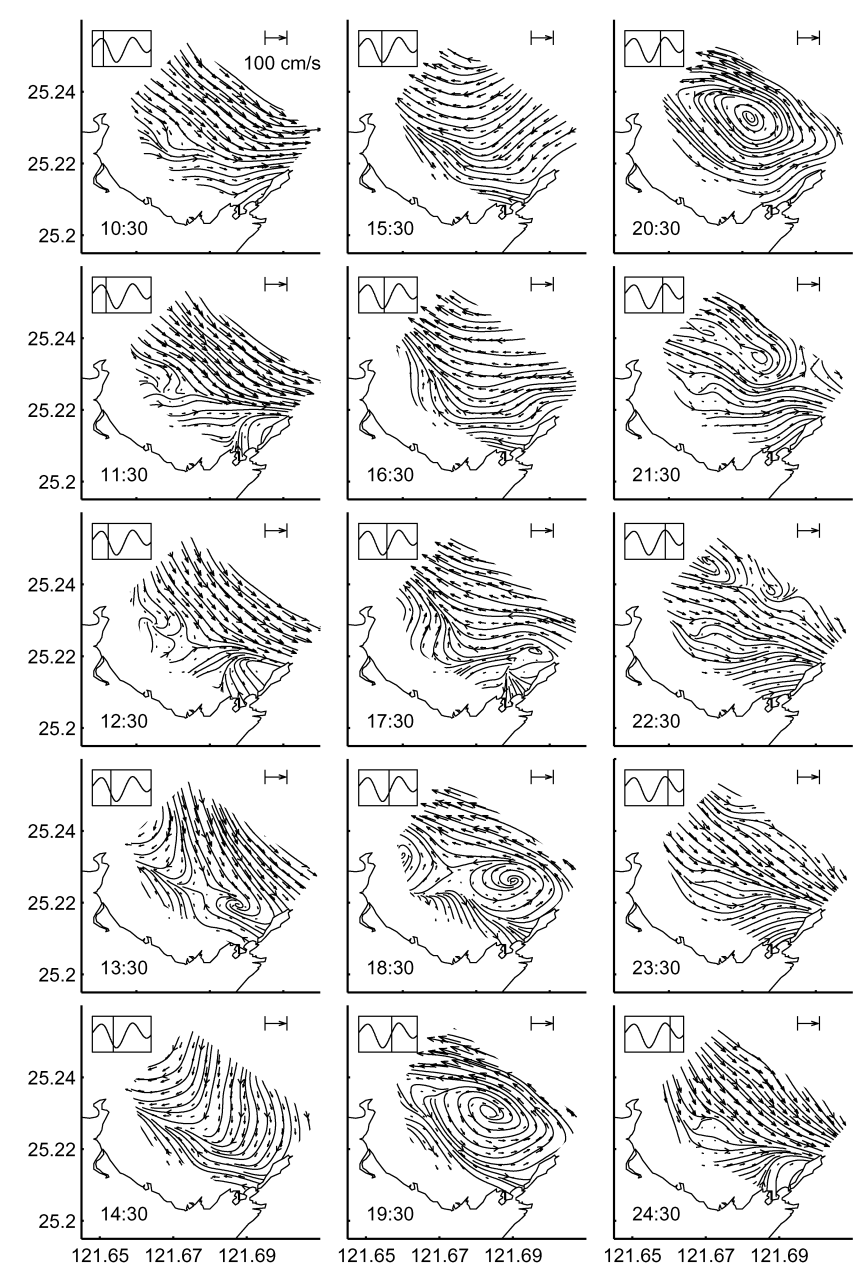

Fig. 8. Synoptic flow charts in vectors and streamlines from SB-ADCP surveys at NPP2 vs. local tide. Time of each frame is in Julian hour since 2001/8/31. 
Gongliao-Fuloon Beach at north of the river mouth. At the northern end of the beach, some reefs spread near the coast and the NPP4 is under construction right there on the shore. From there, rocky coast extends northward to Bito Cape as that of entire Santiao Peninsula, allowing some small ports existed. Such a coastal geography agrees with the early current observations by Lagrangian GPS drifters that waters near the sand beach almost always drifted northward in all kinds of tides (Hwang et al., 2002).

In 2003, the $500 \mathrm{~m}$ pear for downloading the heavy units of the nuclear reactor from sea to NPP4 was finished, the current field along the beach then became so important to estimate if the amount of sand on beach was diminishing, an issue the environment protectors strongly criticized. Since the size of the beach is less than one fifth of the total coastal line and the region of water movement regarding the sand transportation is so
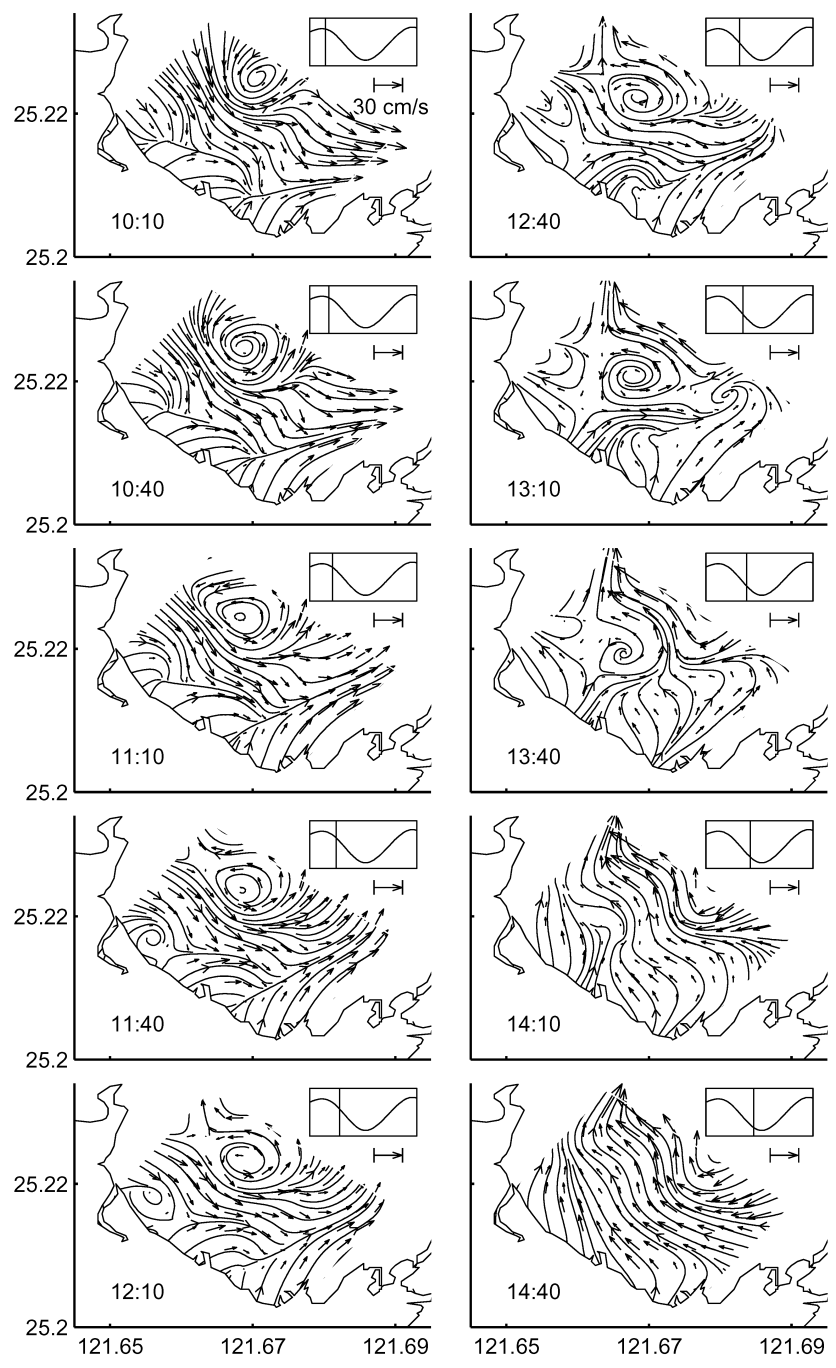

Fig. 9. Near shore synoptic flow charts in vectors and streamlines from ADP surveys at NPP2 on 2001/8/31 vs. local tide. narrow and closed to the beach, to study the current field of the entire bay would cover what needed for such a topic. Notwithstanding, what the current field looks like in the bay and how it interacts with currents outside the bay becomes difficult to survey due to the big size of the bay. Namely it is just about possible to do the ADCP scan over the bay area without knowing the far field at present.

The series of synoptic flow charts in Fig. 11 was obtained from the RV/OR2 ADCP observations in May of 2004. It is obviously seen that those vectors near the Gongliao-Fuloon Beach were dominated by northward flows. For the few exceptions, it should be pointed out that the desired currents very closed to the beach were actually unattainable in Fig. 11 due to the $16 \mathrm{~m}$ blank zone of the SB-ADCP system. As to whether a vortex was initiated inside the bay and then bounced to the open sea as in previous cases, the one developed at south of Bito Cape since 17:00 was quite rigid and expanded in soon to affect about the entire bay as an anti-cyclonic gyre. This gyre having a size of the bay mouth was also observed right there by the coastal radar system, CODAR (Hu, 2004). Notwithstanding, it should be noted that the ebb currents outside the bay mouth were much stronger than flood currents due to the turning effect upon the Cape Bito (see Fig. 1). Therefore the alternation of ebb to flood currents had a time lag to tide with very significant speeds around the low tide. To the contrary, the current field became vague or weak right during the slack time of high tide. The mixed type of tides one day before and after the experiment date is drawn in Fig. $11 \mathrm{~b}$, indicating that the low tide lower than the mean sea level was more than what of high tide, another possible cause of the significant ebb currents.

On the other hand, there seemed no vortex induced by Santiao Cape at all. As a matter of fact, careful

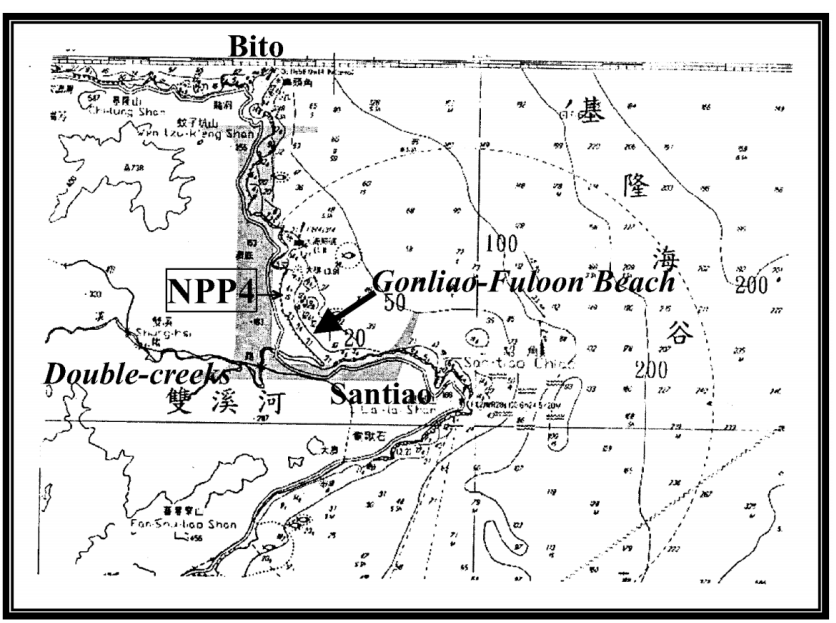

Fig. 10. The bottom topography at Santiao Bay where the NPP4 located. 


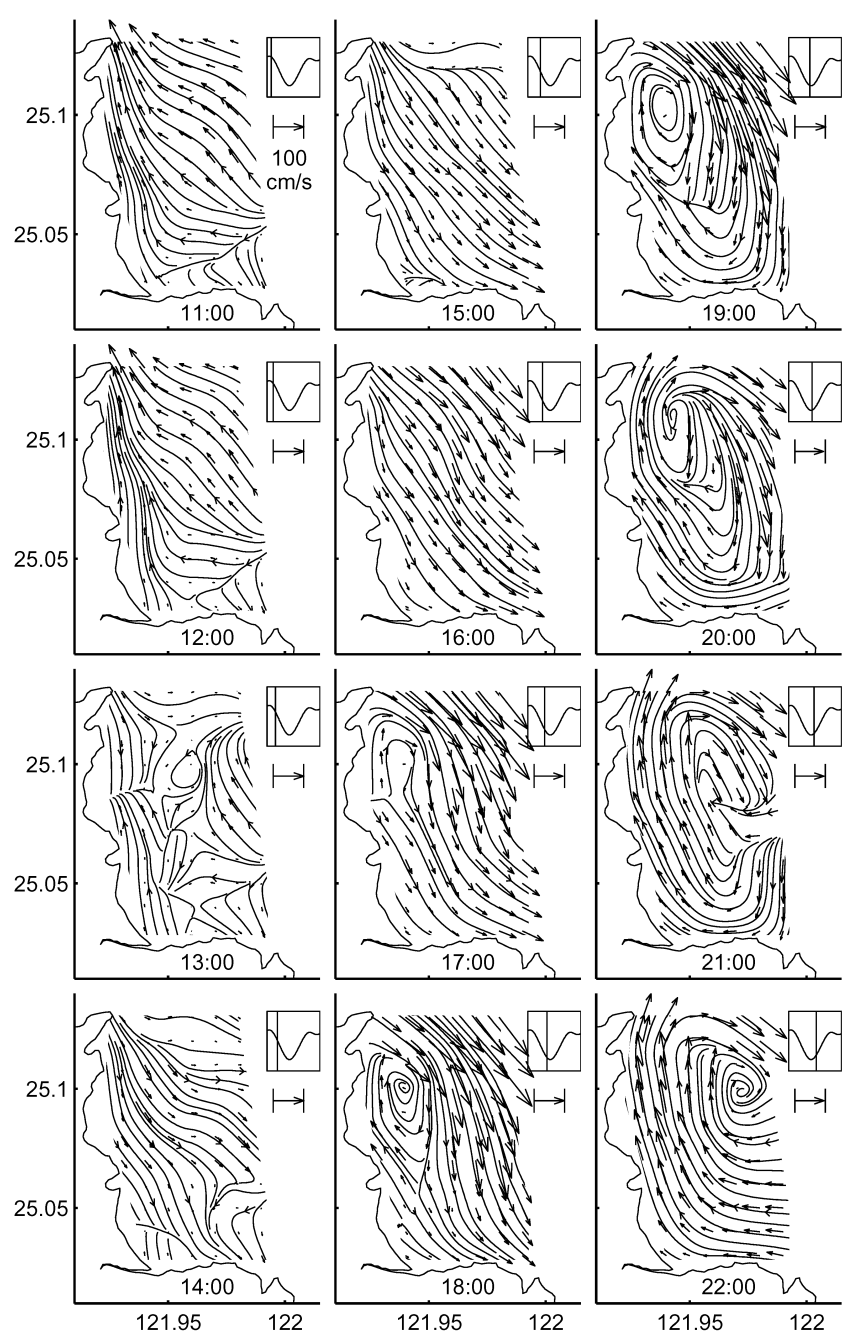

(a)
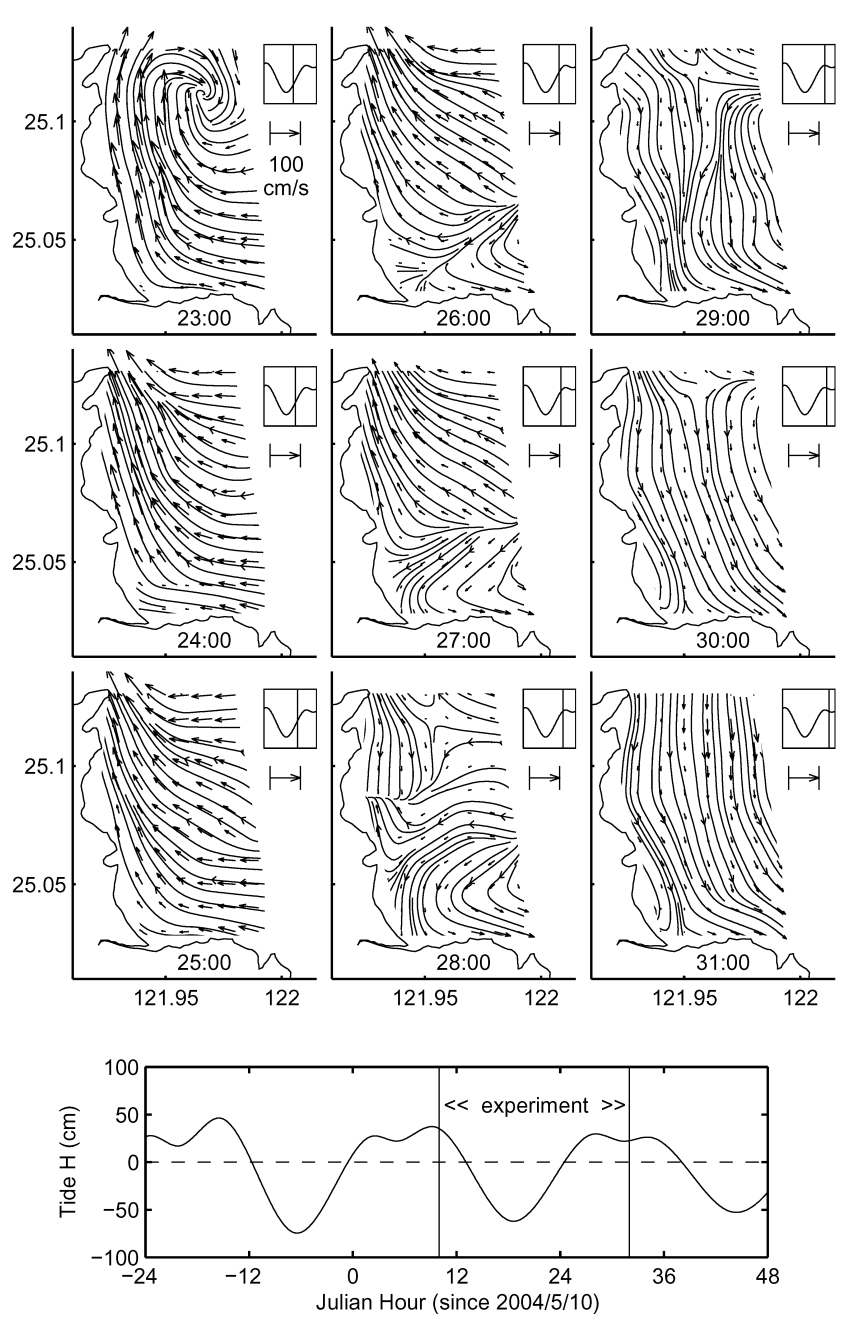

(b)

Fig. 11. (a) Synoptic flow charts in vectors and streamlines from SB-ADCP surveys at NPP4 vs. local tide. Time of each frame is in Julian hour since 2004/5/10. (b) Continued of (a). The bottom graph is tide around 2004/5/10.

inspection may detect the small and weak cyclonic vortices near the tip of Santiao Cape, such as those at 12:00-14:00 and 26:00-28:00. They had no chance to get growing because the bay mouth is too wide to allow a meandering like intrusion to the center of the bay. Therefore it could be rather to view the GongliaoFuloon coastal waters mainly influenced by the two capes than a bay.

\section{SUMMARY AND CONCLUSION}

There are three nuclear power plants (NPP) at northern Taiwan, the NPP1, NPP2 and NPP4. The former twos have been running for more than twenty years, while the third is still under construction. In order to monitor the environments of where the thermal plumes have or will affect, a long term surveys on the coastal ecosystems near the plants have been conducting since beginning, in which the coastal currents are basic mechanism to disperse the warm water and thereby since ever the first aim to be investigated. In early years, the currents were observed with traditional methods of current meter moorings and drifters, while the warm waters were sampled by several hydrographic stations around the discharged channel. Analyses of those data were reported with inevitable tidal phenomenon and not much else. In fact, more devices employed would have turned out more difficult to elucidate the dynamic field.

In the recent years, the modern device SB-ADCP provided a powerful technique to scan over a water area for an observation of the three-dimension velocity field. 
This idea is usually good for open seas, but not quite so straightforward to apply for a coastal area of complex, for instance, the bay or area with capes and peninsula etc.. The coastal geography at NPP1 is characterized by a subsurface valley extended from shore. On top of the east slope of the valley a breakwater normal to the coastline was built to form a "quasi-bay" of NPP1. The NPP2 resides at Yelio Bay and the NPP4 at Santiao Bay. Therefore, to analyze the ADCP scanned data requires certain process to obtain a time series of synoptic flow charts. And then the current fields become so clear for interpretation and well ready for further studies.

To reveal the tidal components of coastal currents from this method is not surprising, but it would be remarkable to disclose how the currents alternate in tides. For coastline with a bay bounded by two capes, currents outside the bay might induce vortices against the capes in cyclonic or anti-cyclonic mode depending upon during flood or ebb tide. After well formed inside the bay in few hours, these vortices would generally bounce out of the bay to trigger or accompany with the alternation of tidal currents over there. At NPP1, the migration of such a vortex might reach a great distance from shore but not for NPP2 and NPP4. At NPP2, the vortex may not move far away from the bay mouth where the bank probably plays a role to dissipate its energy. At NPP4, the bay is too big with a too wide mouth to exhibit its effect on the coastal currents as others. A rather large anti-cyclonic vortex may be generated near Bito Cape and perhaps developed to have a size of the bay mouth, consistently describing how the coastal tidal currents response to the boundary layer effects.

\section{REFERENCES}

1. Candela, J., Beardsley, R.C., and Limeburner, R., "Separation of Tidal and Subtidal Currents in Ship-mounted Dopper Current Profiler Observations," J. Geophy. Res., Vol. 97, C1, pp. 769-788 (1992).

2. Chang, C.-H., "The Relation Between Coastal Currents and Bay Shapes at Northern Taiwan-Case Study on the Thermal Plume of Nuclear Power Plants," M.S. Thesis, Dept. of Oceanography, National Taiwan Ocean University, Keelung, Taiwan (2002) (in Chinese).

3. Chen, J.-F., "The Response of 3D Hydrographic Fields to Tidal Currents at Keelungyu Sill in Summer Time," M.S. Thesis, Dept. of Oceanography, National Taiwan Ocean University, Keelung, Taiwan (2004) (in Chinese).

4. Chen, S.-Y., "Upon the Variation of Tidal Currents-Case Study of Keelung Coastal Area," M.S. Thesis, Dept. of Oceanography, National Taiwan Ocean University, Keelung, Taiwan (2002) (in Chinese).

5. Hu, J.-H., "On the Keweenaw Current: Visual and Numerical Studies of the Mean and Turbulent Flows," $\mathrm{Ph}$. D. Dissertation, University of Wisconsin-Madison, Madison, WI (1983).

6. Hu, J.-H. and Fang, T.-H., "On the Physical and Chemical Environment near the Coasts of Nuclear Power Plants at Northern Taiwan," Report of National Taiwan Ocean University, Keelung, Taiwan (2000) (in Chinese).

7. Hu, J.-H., "The Currents in the Region Affected by Discharged Water of Keelung City Sewage Disposal Plant," Report of National Taiwan Ocean University and Keelung City Government, Keelung, Taiwan (2001a) (in Chinese).

8. Hu, J.-H., The Coastal Tidal Currents near the National Museum of Marine Science and Technology at Bodoji, ROC National Museum of Ma-rine Science and Technology, Report No. 90-03-03, Keelung, Taiwan (2001b) (in Chinese).

9. Hu, J.-H., "The Remote and Scanning Observations of Currents in Coastal Boundary Layer Effective Regions," Symposium of the Workshop on Operational Wave and Current Observation in Taiwan, ROC Central Weather Bureau, Taipei, Taiwan, pp. 37-46 (2004) (in Chinese).

10. Hwang, J.-S., Shao, K.-T., Cheng, I.-J., Fu, C.-A., Hu, J.-H., Fang, T.-H., and Lo, W.-T., "The Ecological Survey of Nuclear Power Plant I, II and Pre-Operation IV, Northern Taiwan," Report of National Taiwan Ocean University, Keelung, Taiwan (2002) (in Chinese). 